Check for updates

NHS Providers

Cite this as: $B M J$ 2022;376:0140 http://dx.doi.org/10.1136/bmj.0140 Published: 18 January 2022

\title{
We must keep recognising the extraordinary efforts of NHS staff
}

\section{Miriam Deakin director of policy and strategy}

Last Thursday, NHS England and NHS Improvement published the latest monthly performance figures and sixth week of winter sitreps. This time, the monthly data provides an invaluable insight into the pressures on the health sector, ahead of the omicron surge, as well as into ongoing seasonal pressures, covid-19 care needs, and the delivery of the vaccination programme, alongside increased demand. While the data highlighted the intense pressures that NHS trusts are under on multiple fronts, it also demonstrated high levels of activity and the incredible efforts of NHS staff to deliver care.

All eyes are on the covid-19 caseload and the impact this is having on capacity across hospitals, ambulances, community, and mental health trusts. Although there are some regional variations, fortunately, the data showed that covid-19 cases seem to be decreasing nationally, with a $30 \%$ fall since the week before. While the covid-19 situation appears to be stabilising across the country, we are still seeing increases in hospital admissions and the total number of patients in hospital in every region. Looking at previous trends however, it is likely to be some weeks until we see the decline in associated pressures across hospitals.

The continued strain across urgent and emergency care continues to be a cause for concern. December's data showed a record-breaking number of the most serious category 1 ambulance calls, and an increase in the number of NHS 111 calls. Emergency department attendances were $27 \%$ higher than the same time last year, and there was an increase in those waiting 12 hours or more for admission in emergency departments. The number of these long waits tripled in a year and are now over five times greater than two years ago before the pandemic.

The winter data for early January showed bed occupancy had increased to $91.8 \%$. This is particularly concerning given the additional demands of covid-19 pathways and the ongoing omicron variant. The number of beds occupied in adult critical care wards have also increased since last week, but thankfully remain lower than the same time last year. Meanwhile the number of beds occupied by flu patients has also increased this week, both overall and in critical care.

The number of delayed discharges continues to grow, given the limitations on capacity in social care as some care homes reduced new admissions due to the rise of omicron-and due to wider pressures on NHS community services. The winter data show there were over 17000 patients who no longer met the criteria to reside in hospital, but $72 \%$ of these remained in hospital. There were also more patients staying longer than seven, 14, and 21 days this week indicating a slowing down of patient flow through the health and care system.
These figures add up to a highly concerning picture, particularly when performance against official targets is factored into the equation, such as the four-hour A\&E waiting time target which now sits at $73.3 \%$ against the standard of $95 \%$. However, there must also be focus on the extraordinary work NHS staff are doing to treat patients within this context of rising operational pressure. For example, while the elective waiting list increased slightly in November to almost six million, the number of admitted inpatient pathways and non-admitted pathways that started that month all increased, showing a rise in activity ahead of the omicron surge. November also saw record numbers of patients seen by a consultant following an urgent two-week GP referral, and all activity in cancer care increased from the previous month. Diagnostic activity reached the highest level since January 2020. This is testament to the hard work of trusts and frontline staff.

But this effort is now taking place against a backdrop of increasing staff absences, which grew by a further $10 \%$ this week and of which over half were due to covid-19. The fact that absence rates are high across all regions in England reflects the movement of omicron beyond the epicentre in London. Trusts have welcomed the support of colleagues from the armed forces in some areas, to help plug some of these staffing gaps. The NHS has worked closely with the military throughout the pandemic, with the rollout of the vaccination programme and support for ambulance services, as notable examples where collaboration has been invaluable.

Trusts undoubtedly have many immediate challenges ahead and trust leaders are rightfully concerned about the strain on all services. We anticipate the data in the coming weeks will continue to show how difficult operational pressures on the service are, with the NHS stretched further than ever before. However, while the data may paint a more concerning picture of performances against official targets, it is our duty, both as organisations and as individuals, to keep banging the drum about the extraordinary volume of activity that staff are succeeding in delivering under the most challenging of circumstances.

Competing interests: none declared

Provenance and peer review: commissioned, not peer reviewed 